\title{
L'élision variable du schwa en fin de mot chez des hommes méridionaux : L'effet des consonnes environnantes et de la fréquence de la lexie
}

\author{
Ranson, Diana L. \& Passarello, Matthew D. \\ Université de Géorgie \\ dranson@uga.edu; mattpass@uga.edu
}

\section{La variabilité du schwa}

La prononciation d'un schwa, appelé aussi $e$ caduc ou $e$ muet, à la fin d'un mot est une caractéristique bien connue des locuteurs du sud de la France, comme le témoignent des manuels d'initiation à la prononciation ou à la linguistique française (Tranel $1987: 105$, Valdman 1993 :51, Fagyal et al. $2006: 60$ ). Ce qui est moins connu, pourtant, est que la prononciation de ce schwa méridional est variable, fait omis par ces mêmes manuels mais bien démontré par les études spécialisées sur ce phénomène (Diller 1978; Durand et al. 1987; Taylor 1996; Armstrong et Unsworth 1999 ; Eychenne 2006 ; Pustka 2007, 2009 ; Durand 2009). Un locuteur méridional peut dire tantôt [koməsa] tantôt [kəmsa] pour comme ça, par exemple, comme le fait le Locuteur B du corpus pour cette étude. Les études antérieures sur la variabilité du schwa méridional considèrent surtout des facteurs diastratiques pour montrer l'effet de la classe sociale (Diller 1978, Taylor 1996), de l'âge (Durand et al. 1987, Taylor 1996), et du sexe (Armstrong et Unsworth 1999) du locuteur sur la réalisation du schwa. Par conséquent, les facteurs linguistiques qui influencent la variabilité de cette voyelle ne sont pas aussi bien connus pour le schwa méridional que pour le schwa dans d'autres positions du mot et dans d'autres régions géographiques. Malécot (1976) et Hansen (1994) pour le dialecte parisien et Racine et Grosjean (2002) pour le dialecte suisse romand montrent que la réalisation du schwa varie non seulement selon des facteurs diastratiques, mais aussi selon des facteurs linguistiques tels que les consonnes qui l'entourent et la lexie qui le contient. Notre but dans la présente étude est donc de complémenter les études antérieures sur le schwa méridional en considérant l'effet des facteurs linguistiques, jusque là inconnu, sur l'élision de cette voyelle. Nous adopterons une approche variationniste labovienne, telle qu'elle est décrite par Tagliamonte (2006), pour déterminer l'effet sur la variabilité du schwa des consonnes qui l'entourent, de la possible interaction entre ces consonnes, et de la fréquence de la lexie dans laquelle le schwa puisse s'élider.

Le choix de ces facteurs linguistiques en particulier se justifie par leur importance dans les études antérieures. Parmi les facteurs qui influencent la réalisation du schwa ce sont les consonnes environnantes qui ont la plus longue histoire. La loi des trois consonnes, formulée par Grammont (1894) et puis raffinée par lui à plusieurs reprises (voir Durand et Laks 2000), maintient qu'un schwa sera réalisé si son élision résultera dans un groupe de trois consonnes. Cela expliquerait, par exemple, pourquoi le schwa serait prononcé en syllabe initiale dans prenons, en syllabe interne dans simplement, en position finale dans énorme maison, ou dans le monosyllabe le dans le principe. Cette notion est une des plus débattues de la phonologie française dont la vaste bibliographie comprend, entre autres, Malécot (1955), Fouché (1959), Pulgram (1961), Dauses (1973), et Spence (1982). Ces nombreuses études sur la loi des trois consonnes visaient un but nettement différent du nôtre, notamment celui de formuler les règles pour décrire précisément dans quels contextes phonologiques le schwa sera obligatoirement prononcé et dans lesquels sa prononciation sera variable, comme le dit Pulgram (1961 :305). Notre but dans la présente étude, par contre, est de déterminer les facteurs qui influencent la variabilité du schwa chez certains locuteurs dans un contexte bien défini, à savoir la position finale de mot entre une seule consonne précédente et une seule consonne ou une pause suivante. Vu nos différents buts, une discussion détaillée de la loi des trois consonnes sorte de notre champ d'étude, mais il est important de remarquer que les phonologues ont toujours considéré les consonnes environnantes comme un point de départ 
essentiel pour l'analyse empirique de l'élision du schwa. Une notion qui sous-tend ces discussions est que le schwa sert de «lubrifiant phonique » (Durand et Laks 2000 :33) qui gère la transition articulatoire entre consonnes. Comme l'a dit Spence (1982:9): «The fact remains surely that the phonotactic (and prosodic) rules of French play the major role in determining the minimum role of schwa as a glide lubricating the flow of consonant clusters in French. » Voilà donc pourquoi il est approprié dans cette première étude variationniste sur les facteurs linguistiques qui influencent l'élision du schwa final méridional de considérer les caractéristiques des consonnes environnantes et l'interaction entre elles.

Plusieurs études sur le schwa dans d'autres dialectes du français que le méridional et dans d'autres positions que la finale proposent que les consonnes environnantes influencent l'élision du schwa. Delattre (1949a :459), d'après son intuition sur la prononciation du schwa des monosyllabes en début d'énoncé, propose qu'une consonne précédente «plus forte-fermée » favorise le maintien du schwa et que, inversement, une consonne précédente plus faible et ouverte favorise son élision. En combinant la liste pour l'aperture des consonnes avec celle pour leur force d'articulation, il propose l'ordre suivant des consonnes de la plus faible-ouverte jusqu'à la plus forte-fermée illustré par les monosyllabes qui commencent par ces consonnes : je, ce-se, le, ne, me, de, te, que. Cela veut dire donc que la consonne / 3 / favorise l'élision du schwa le plus et que le / $\mathrm{k} /$ le défavorise le plus. Dans la suite de cet article, Delattre (1949b:44) propose en plus une interaction entre les consonnes autour du schwa dans une suite de deux monosyllabes en début d'énoncé, comme que je ou je te ou de ce. Une première consonne moins forte-fermée (ou plus faible-ouverte) que la seconde favoriserait l'élision du schwa de façon à ce que je te soit prononcé le plus souvent comme $j$ te [jtə] tandis qu'une première consonne plus forte-fermée favoriserait le maintien du schwa de façon à ce que que je soit prononcé le plus souvent comme que j [kəz]. Malécot (1976), suivant Delattre (1949b), teste empiriquement l'interaction entre consonnes selon leur force dans son analyse de 10.891 occurrences de schwa dans des monosyllabes en début d'énoncé dans le dialecte parisien. Comme Delattre (1949b), Malécot (1976:98-99) propose qu'un schwa précédé par une consonne faible et suivi par une consonne forte sera plus souvent élidé, comme le schwa de je dans je pense, et qu'un schwa aura tendance à être réalisé s'il est précédé par une consonne forte et suivi par une consonne faible, comme le schwa de que dans Que voulez-vous? Parmi les schwas en début d'énoncé dans son corpus, seulement $4 \%$ (2/47) de ceux qui suivent une occlusive, la consonne la plus forte, sont élidés contre 44\% (24/54) de ceux qui suivent une fricative, une consonne plus faible que l'occlusive. Mais le schwa de le, le seul mot monosyllabique qui commence par $/ 1 / \mathrm{n}$ 'est jamais élidé dans ce corpus (24/24).

D'après les études empiriques de Hansen (1994) et Racine et Grosjean (2002), l'effet de la force consonantique semble être l'inverse quand le schwa se trouve en syllabe initiale de mot. Hansen (1994:44) trouve dans son corpus parisien que le schwa dans la syllabe initiale des mots, quand on exclut l'initiale en $r e$-, a tendance à être réalisé s'il est précédé d'une consonne faible et suivi d'une consonne forte, le contraire de ce qu'on vient de voir pour les monosyllabes en début d'énoncé. Le taux de maintien du schwa est de $68 \%$ quand la première consonne est plus faible que la seconde, comme dans depuis, et de $26 \%$ seulement quand la première consonne est plus forte, comme dans demi. Pour la syllabe initiale re- la force articulatoire proposée par Delattre (1949b) et Malécot (1976) pour les monosyllabes est «partiellement valable» (Hansen 1994 :44). Dans les mots qui commencent par re- la consonne qui précède le schwa est toujours $/ \mathrm{R} /$, une consonne faible, et donc la force relative des consonnes environnantes dépend de la consonne suivante. Plus la consonne qui suit le schwa est forte, ce qui veut dire que la séquence des consonnes environnantes va de consonne faible à consonne forte, plus le schwa est élidé. Donc dans ces mots, une consonne précédente faible suivie par une consonne plus forte favorise l'élision du schwa, comme le disent Delattre (1949b :44) et Malécot (1976:99) pour les monosyllabes en début d'énoncé.

Racine et Grosjean (2002) testent sept facteurs pour la prononciation du schwa dans la syllabe initiale de 66 mots chez 16 locutrices suisses romandes dont deux sont relatifs au contexte phonologique. Ils reproduisent le test de la force articulatoire de Hansen (1994), mais ils considèrent 
le degré de force, plutôt que la force relative, en attribuant une valeur de 5 aux occlusives sourdes, de 4 aux occlusives sonores, /b/, /d/, /g/, de 3 aux fricatives sourdes, /f/, /s/, / / /, de 2 aux fricatives sonores, de 2 aux fricatives sonores, /v/,/z/,/3/, et de 1 aux consonnes les plus faibles, /m/, /n/, /r/, et /1/. Pourtant l'effet de ce facteur sur l'élision du schwa n'est pas significatif pour la syllabe initiale de mot (Racine et Grosjean 2002 :316-317). Ces auteurs établissent aussi un moyen de tester l'effet de l'environnement consonantique sur la réalisation du schwa qui tient compte du nombre de consonnes environnantes et de leur valeur sur "l'échelle universelle de sonorité " (Racine et Grosjean 2002 :314-315). Clements (1990:292) établit l'ordre de sonorité suivant de la plus haute sonorité à la plus basse : les voyelles, les semi-voyelles, les liquides, les nasles, les obstruantes. Racine et Grosjean (2002:315) comptent le nombre de consonnes avant et après le schwa et puis ils ajoutent un point si la sonorité est descendante, c'est-à-dire si elle va d'une consonne plus faible à une consonne plus forte, et ils soustraient un point pour la sonorité ascendante qui va de consonne plus forte à consonne plus faible, puisqu'ils considèrent cette transition comme une prononciation « relativement aisée » (Racine et Grosjean 2002 :315). Selon cette formule, une valeur plus basse favorisera l'élision du schwa tandis qu'une valeur plus haute favorisera son maintien. Ils attribuent, par exemple, une valeur très basse de 1,0 à semaine qui a un taux d'élision de schwa de $94 \%$. Le mot semaine gagne 2 points pour les deux consonnes environnantes et perd un point pour la sonorité ascendante entre $/ \mathrm{s} /$ et $/ \mathrm{m} /$. Alors on peut constater que Racine et Grosjean considèrent qu'un plus petit nombre de consonnes favorisera l'élision du schwa ainsi que la sonorité ascendante pour la syllabe initiale, ce qui va à l'encontre de l'idée de Delattre (1949b :44) et Malécot (1976:99) pour les monosyllabes en début d'énoncé que le schwa sera élidé si la sonorité est descendante, c'est-àdire si la première consonne est plus faible que la seconde. Racine et Grosjean $(2002: 315)$ trouvent que l'environnement consonantique a un effet significatif sur le maintien du schwa et en tirent la conclusion que «plus le groupe consonantique en contact est important et difficile à prononcer, moins ce mot va être produit avec effacement. » Ils ont choisi de combiner la taille du groupe consonantique avec le degré de sonorité puisque la taille du groupe consonantique toute seule s'est avérée insignifiante. Pourtant cette décision de codage fait que nous ne savons pas si le degré de sonorité aurait été significatif à lui seul.

Les études antérieures sur la force des consonnes environnantes suggèrent donc une tendance opposée dans les monosyllabes (Delattre 1949b, Malécot 1976) et dans la syllabe initiale de mot (Hansen 1994, Racine et Grosjean 2002). Une consonne précédente plus forte que celle qui suit le schwa potentiel défavorise l'élision du schwa dans les monosyllabes mais favorise son élision en syllabe initiale, sauf en initiale en re-. Il sera intéressant donc de voir si les consonnes environnantes ont un effet sur l'élision du schwa en fin de mot et, si c'est le cas, si leur effet suivra la tendance observée pour les monosyllabes ou pour les syllabes initiales.

En plus de l'effet des consonnes environnantes sur l'élision du schwa, Hansen (1994) et Racine et Grosjean (2002) ont identifié un autre facteur linguistique que nous testerons dans la présente étude : la fréquence de la lexie dans laquelle le schwa puisse apparaître. Hansen (1994 :43), qui détermine la fréquence des lexies à partir de leur nombre d'occurrences dans son corpus de français parisien parlé et par deux autres mesures, trouve que la fréquence de la lexie ne joue aucun rôle significatif pour les mots sans re- à cause de la grande hétérogénéité de ce groupe de mots. Pourtant, pour les mots en $r e$ - «la fréquence exerce une influence statistiquement valable sur le maintien du $\mathrm{E}$ caduc » (Hansen 1994 :43). Plus un mot en re- est fréquent, plus le schwa est élidé. Racine et Grosjean (2002), comme le fait Hansen (1994) pour les mots en re-, trouvent une corrélation positive entre la fréquence de la lexie et l'élision du schwa. Mais comme leur étude était basée sur la production guidée des récits et non pas sur des conversations spontanées, ils ont déterminé la fréquence des lexies en demandant à 14 locuteurs de la Suisse romande, qui n'ont pas participé à la tâche de production, d'estimer la fréquence des 66 mots cibles dans leur étude. Walker (1996) dans une étude sur la réalisation du schwa en syllabe initiale dans 1.250 lexies dont la prononciation était fournie par quatre dictionnaires de prononciation souligne aussi le rôle important des lexies pour la 
réalisation du schwa mais sans associer l'effet de la lexie sur le maintien du schwa avec sa fréquence.

Puisque notre but dans la présente étude est de tester l'effet des facteurs linguistiques sur l'élision du schwa, il est nécessaire pour isoler ces facteurs de garder constants les facteurs diastratiques qui puissent influencer ce phénomène. Durand et al. (1987), dans leur étude sur le schwa chez quatre locutrices d'une même famille de Pézenas dans le département de l'Hérault, notent l'élision du schwa dans toutes les positions : syllabe initiale de mot, syllabe interne, monosyllabe, fin de mot devant pause, fin de mot devant consonne, et dans des expressions figées. Pour la position finale de mot, celle qui nous intéresse ici, ils trouvent que seulement la locutrice la plus jeune élide le schwa devant pause et que le taux d'élision en position finale devant consonne est plus élevé plus la locutrice est jeune. Le taux d'élision est de $0 \%$ pour la locutrice de 85 ans, de 3,6\% pour celle de 67 ans, de $16,7 \%$ pour celle de 36 ans, et de $47,5 \%$ pour la plus jeune locutrice âgée de 16 ans. Taylor (1996 :162, 297), dans son étude sur la parole de 59 locuteurs aixois, et Pustka (2007:85-86), dans une étude sur la parole de 10 locuteurs aveyronnais, trouvent également que les plus jeunes locuteurs affichent en général un taux d'élision du schwa plus haut que celui des locuteurs les plus âgés. Il faut noter pourtant que dans l'étude de Taylor cette différence a manqué d'atteindre le seuil de significativité ( $\mathrm{p}=0.0513)$ et que les taux d'élision ont été calculés pour le schwa en monosyllabe, en syllabe initiale de mot, après deux consonnes, après une seule consonne, et dans toutes les positions confondues, mais pas pour le schwa en fin de mot. Puisqu'il est clair que l'âge a un effet sur la réalisation du schwa, nous avons décidé de limiter notre corpus aux locuteurs âgés de plus de 45 ans. Ce choix est basé en plus sur une considération pratique. Les jeunes locuteurs de notre corpus, recueilli au moins 18 ans après celui de Pézenas, élident presque toujours le schwa en fin de mot et donc leur parole ne se prête pas à une analyse variationniste du phénomène. En ce qui concerne le sexe, Armstrong et Unsworth (1999), dans une étude de la parole de 16 jeunes locuteurs âgés de 16 à 25 ans de Carcassonne et Lézignan-Corbières dans le département de l'Aude, trouvent que les femmes élident le schwa plus souvent que les hommes. En fin de mot devant pause le taux d'élision chez les femmes est de 43,7\% contre 18,5\% chez les hommes et le taux d'élision du schwa final devant consonne est encore plus élevé : $63,4 \%$ chez les femmes contre $40,4 \%$ chez les hommes. Taylor (1996:162), par contre, n'a trouvé aucune différence entre les sexes, mais cela se doit possiblement au fait qu'elle n'a pas isolé le schwa en fin de mot. Vu l'effet possible du sexe du locuteur sur l'élision du schwa final, nous avons choisi d'analyser la parole des hommes seulement pour ne pas introduire le sexe comme variable dans l'étude. Puisque notre corpus ne contient pas un nombre suffisant de locuteurs qui se distinguent uniquement par la classe sociale, comme nous le verrons dans le Tableau 2 ci-dessous, nous n'avons pas pu isoler la classe sociale des locuteurs, signalée comme importante par Diller (1978) et Taylor (1996). Diller (1978:17-18) trouve que le schwa en fin de mot chez dix hommes âgés de 55 à 70 ans de la province de Béarn est plus souvent élidé par les hommes qui ont fait des études universitaires que par ceux qui n'ont fait que l'école primaire. De la même façon Taylor $(1996: 158-60,288,291)$ trouve un plus haut taux d'élision du schwa chez les locuteurs les plus instruits et ceux qui exercent une profession libérale plutôt que manuelle.

\section{Méthodes}

\subsection{Corpus}

La présente étude analyse la parole de dix hommes du sud de la France âgés de 45 à 73 ans, avec un âge moyen de 55 ans. Le Tableau 1 présente l'identifiant de chaque locuteur ainsi que son âge, son niveau d'éducation et son lieu d'origine. Quatre locuteurs font partie du Projet de la Phonologie du Français Contemporain (Projet PFC) et leur identifiant pour ce projet se trouve entre parenthèses après leur identifiant pour la présente étude. Huit locuteurs du corpus étaient originaires du département des Bouches du Rhône. Parmi eux, quatre étaient enregistrés par la première auteure de 
cette étude en 2006 à Rognes, une ville située à 20 kilomètres au nord-ouest d'Aix-en-Provence. Deux y avaient passé toute la vie tandis qu'un autre était originaire de Pélissanne, une ville à 20 kilomètres à l'ouest de Rognes, où il avait toujours habité. Le quatrième avant grandi à Martigues avant de déménager à Rognes. Quatre autres locuteurs qui venaient aussi du département des Bouches du Rhône avaient été enregistrés entre 2002 et 2004 pour les enquêtes Aix-Marseille et Marseille centre ville du Projet PFC. Tous les quatre étaient nés à Marseille; trois y avaient passé toute la vie et le quatrième habitaient près d'Aix-en-Provence. Deux autres locuteurs habitaient à Montpellier au moment de leur interview en 2005 par la première auteure dont un était originaire de Béziers dans le département de l'Hérault et l'autre de Conques dans le département voisinant de l'Aveyron. Comme nous l'avons dit ci-dessus, nous avons choisi d'analyser la parole des locuteurs plus âgés parce que les locuteurs plus jeunes dans ce corpus, même ceux du sud, ne prononçaient que rarement le schwa en fin de mot et donc n'affichaient pas une prononciation variable de cette voyelle. La décision d'analyser des locuteurs du même sexe visait à ne pas introduire le sexe comme facteur dans une analyse de l'effet des facteurs linguistiques sur l'élision du schwa. Nous avons choisi les hommes parce qu'il était notre impression en écoutant les enregistrements qu'ils élidaient moins souvent le schwa que les femmes, ce qui était le cas aussi dans l'étude d'Armstrong et Unsworth (1999), et qu'ils avaient donc une prononciation plus variable de cette voyelle. Il y a parmi les locuteurs une différence en ce qui concerne le niveau d'études. Deux d'entre eux n'ont pas fini le baccalauréat (Locuteurs $\mathrm{C}$ et $\mathrm{F}$ ), cinq ont fait comme minimum le bac (Locuteurs $\mathrm{A}, \mathrm{B}, \mathrm{D}, \mathrm{H}$ et $\mathrm{J}$ ), et trois ont fait le bac plus un minimum de quatre ans d'études (Locuteurs E, G, I). Nous verrons dans le Tableau 2 ci-dessous que le taux d'élision du schwa pour chaque locuteur ne semble pas correspondre à son niveau d'études, mais nous avons voulu constater ces différences.

Tableau 1 : L'âge, le niveau d'études, et le lieu d'origine des locuteurs de la présente étude

\begin{tabular}{|l|l|l|l|}
\hline locuteur & âge & niveau d'études & lieu d'origine \\
\hline A & 45 & bac & Pélissanne \\
\hline B (13brp2) & 45 & bac ou bac +2 & Marseille \\
\hline C & 54 & sans bac & Rognes \\
\hline D & 54 & bac +2 & Martigues \\
\hline E (13apd1) & 54 & bac +4 & Marseille \\
\hline F & 55 & sans bac & Rognes \\
\hline G & 56 & bac +5 & Béziers \\
\hline H (13bpa1) & 58 & bac & Châteauneuf le Rouge (près d'Aix) \\
\hline I & 65 & bac +5 & Conques \\
\hline J (13aas1) & 73 & bac & Marseille \\
\hline
\end{tabular}

\subsection{Analyse}

Dans un premier temps, nous avons noté sur la transcription écrite des conversations de 5 minutes par locuteur tous les schwas en fin de mot précédés par une seule consonne et suivis par une seule consonne ou une pause. Le choix de baser l'identification des schwas potentiels sur le texte écrit est soutenu par la constatation de Durand et al. (1987:986) que l'orthographe représente assez bien la présence d'un schwa dans cette position. Nous avons exclu les mots où le schwa est précédé ou suivi par une voyelle parce que le schwa n'est jamais prononcé devant ou après voyelle dans notre corpus ni dans celui de Durand et al. (1987 :986). Nous avons exclu en plus les mots où le schwa est précédé ou suivi par deux consonnes ou plus puisque, comme le maintient la loi des trois consonnes, un plus grand nombre de consonnes défavorise l'élision du schwa et pourrait donc affecter l'analyse de l'effet des consonnes environnantes sur l'élision du schwa. Nous avons recueilli ainsi 523 occurrences de schwa en fin de mot précédé d'une seule consonne et suivi par une seule consonne ou une pause. 
Dans un deuxième temps nous avons écouté les enregistrements des conversations pour déterminer si les locuteurs avaient prononcé ou élidé le schwa en question. Cette décision est parfois rendu difficile par la ressemblance entre le schwa et tois autres voyelles. D'une part, le schwa ressemble à la voyelle brève qui résulte de la détente d'une occlusive sourde comme dans le mot toute prononcé comme [tut ${ }^{2}$ ] (Valdman $1993: 51$, voir aussi Durand et al. 1987 :990). Valdman (1993:51) signale qu'«il ne faut pas confondre cette voyelle finale brève avec la voyelle pleine [ə], qui caractérise l'accent dit méridional. Cette voyelle a pleine valeur syllabique au même titre que les autres voyelles de la langue ... ». Nous avons choisi de nous appuyer sur la durée de ces voyelles pour distinguer entre les deux. Avec l'aide du logiciel TF32 nous avons établi une durée minimum de $50 \mathrm{~ms}$ pour la réalisation du schwa. Nous avons donc mesuré la durée des cas pour lesquels nous hésitions entre une classification comme schwa et comme voyelle brève de la détente et avons exclu de notre analyse du schwa les voyelles avec une durée plus courte que $50 \mathrm{~ms}$. Cette valeur est en dessous de la durée moyenne de 73,72 ms trouvée par Coquillon et al. (2000 :91) chez les locuteurs marseillais. Le schwa peut aussi se confondre avec la voyelle d'hésitation euh (Durand et al. 1987 :990). Nous n'avons pas identifié de durée maximum du schwa, mais nous avons pris soin d'exclure les cas où la voyelle semblait se prolonger pour indiquer une hésitation. Nous avons aussi exclu les schwas dans les dates, puisque le mot [mil], qui peut s'écrire come mil ou mille, ne termine pas forcément par un schwa et parce que le schwa à la fin des mots indiquant les décennies, comme cinquante et soixante, est toujours maintenu par les locuteurs du corpus dans les dates comme cinquante-quatre et soixante-neuf (voir aussi Durand et al. 1987 :989). Une troisième voyelle qui se prononce de façon similaire au schwa méridional est le $e$ prépausal non étymologique attesté dans le parler parisien (Hansen 1997, Fagyal 2000, Hansen et Hansen 2002), comme à la fin du mot bonjour. Pourtant nous n'avons pas noté de schwas non étymologiques dans notre corpus. En plus, après que nous montrerons que le taux d'élision du schwa est plus bas en position prépausale qu'en position préconsonantique, nous nous concentrerons sur la prononciation du schwa devant consonne et écarterons la position prépausale des analyses.

Une fois notés tous les exemples des schwas finals dans notre corpus, nous avons classé chaque exemple selon le mode et point d'articulation et le voisement de la consonne qui le précédait et qui le suivait. Pour le mode d'articulation, nous avons classé les consonnes comme occlusives (/p/, /t/, $/ \mathrm{k} /, / \mathrm{b} /, / \mathrm{d} /, / \mathrm{g} /)$, fricatives $(/ \mathrm{f} /, / \mathrm{v} /, / \mathrm{s} /, / \mathrm{z} /, / \mathrm{J} /, / \mathrm{z} /)$, nasales $(/ \mathrm{m} /, / \mathrm{n} /, / \mathrm{j} /)$, la liquide $/ \mathrm{l} /$, et la semivoyelle $/ \mathrm{j} /$. En ce qui concerne le $/ \mathrm{R} /$, nous l'avons classé dans un premier temps comme fricative, mais puisque le taux de maintien de schwa après le / $\mathrm{R} /$ était même plus bas que celui après /1/, nous avons décidé par la suite de le classer plutôt comme liquide, ce qui est aussi la classification proposée par Hansen (1994:44). Les consonnes classées selon le point d'articulation comprennent les bilabiales $(/ \mathrm{p} /, / \mathrm{b} /, / \mathrm{m} /)$ et les labiodentales $(/ \mathrm{f} /, / \mathrm{v} /)$, les dentales $(/ \mathrm{t} /, / \mathrm{d} /, / \mathrm{s} /, / \mathrm{z} /, / \mathrm{l} /)$, les palatales $(/ \mathrm{s} /, / \mathrm{z} /, / \mathrm{n} /, / \mathrm{j} /)$ et les vélaires $(/ \mathrm{k} /, / \mathrm{g} /, / \mathrm{R} /)$. Les consonnes non voisées sont $/ \mathrm{p} /, / \mathrm{t} /, / \mathrm{k} /, / \mathrm{f} /, / \mathrm{s} /, / \mathrm{s} /$ tandis que les voisées sont /b/,/d/, /g/, /v/,/z/,/z/, /R/,/m/,/n/,/n/, /1/, /j/. Le segment suivant peut être une des consonnes mentionnées ci-dessus ou aussi la pause. En plus, nous avons classé les consonnes selon leur force articulatoire selon l'échelle adoptée par Hansen $(1987: 44)$. Les consonnes des plus fortes aux plus faibles sont les occlusives sourdes, /p/, /t/, / k/, les occlusives sonores, /b/, /d/, /g/, les fricatives sourdes, /f/, /s/, / / /, les fricatives sonores, /v/, /z/, /3/, les nasales, $/ \mathrm{m} /$ et $/ \mathrm{n} /$, et les liquides $/ 1 /$ et $/ \mathrm{R} /$. La fréquence des lexies terminant par schwa a été calculée selon leur nombre d'occurrences dans notre corpus au lieu de se baser sur leur fréquence générale dans la langue française.

Pour déterminer le poids de chaque facteur, sa significativité statistique (avec un seuil de significativité de $\mathrm{p}<0,05$ ), et son importance relative, nous avons soumis les données à une analyse multivariée par le biais du logiciel GoldVarb X. Pour effectuer cette analyse il était parfois nécessaire d'exclure des occurrences de schwa si sa prononciation n'était pas variable à l'intérieur d'une certaine catégorie. Comme ces exclusions varient d'une analyse à l'autre, elles seront notées, s'il y en a, après le tableau qui présente les résultats pour cette catégorie. 


\section{Résultats et discussion}

Le taux d'élision du schwa dans notre corpus est relativement élevé par rapport à celui des corpus dans les études de Diller (1978), qui a analysé la parole de 10 hommes béarnais âgés de 55 à 70 ans, de Durand et al. (1987 :997), qui ont analysé la parole de quatre femmes de Pézenas de la même famille âgées de 85, 67, 36 et 16 ans, et d'Armstrong et Unsworth (1999:140-141), qui ont analysé la parole de 16 locuteurs de Carcassonne et de Lézignan-Corbières qui avaient entre 16 et 25 ans. Comme le Tableau 2 le montre, le taux d'élision en fin de mot dans notre corpus est de $65 \%$ tandis que Diller (1978:15) a trouvé un taux d'élision de 35\%, Durand et al. (1987:997) de 11\% et Armstrong et Unsworth $(1999: 140-141)$ de $39 \%$. Une raison possible pour ces différents taux d'élision est l'origine des locuteurs. Huit de nos dix locuteurs viennent de la région Provence-AlpesCôte-D'azur tandis que les locuteurs dans les études de Diller (1978), Durand et al. (1987) et d'Armstrong et Unsworth (1999) viennent de plus à l'ouest de la France. Coquillon et al. (2000) ont montré une différence très nette pour la prononciation du schwa en fin de mot entre les Marseillais et les Toulousains, ces derniers ayant une durée supérieure pour cette voyelle. Mais même nos deux locuteurs qui viennent de Languedoc-Rousillon, les Locuteurs G et I, élident bien plus souvent le schwa que les locuteurs des études antérieures. Une autre explication serait les dix ou vingt ans d'écart entre la date d'enregistrement des sujets parlants dans les études antérieures et la nôtre. Il est possible que l'élision du schwa ait progressé entretemps, mais ces différences sont tout de même très grandes. Une autre explication possible est que, dans notre souci d'écarter la voyelle brève de la détente, nous ayons exclu des schwas que d'autres chercheurs auraient comptés.

Bien que le taux d'élision soit plus élevé dans la présente étude, nous avons trouvé, comme dans les études antérieures, que le taux d'élision est plus bas devant pause que devant consonne. Dans notre corpus le taux d'élision devant pause est de $56 \%$ contre $70 \%$ devant consonne. On trouve un taux d'élision du schwa de $23 \%$ devant pause contre $40 \%$ devant consonne dans l'étude de Diller (1978:15), de $2 \%$ contre $19 \%$ dans l'étude de Durand et al. (1987:997) et de $31 \%$ contre $52 \%$ dans l'étude d'Armstrong et Unsworth (1999:140-141). Vu la différence importante entre le taux d'élision devant pause et celui devant consonne, nous nous concentrerons dans les analyses subséquentes sur le comportement variable de schwa seulement devant consonne pour mieux voir l'effet des consonnes environnantes sur son élision.

Tableau 2 : Élision du schwa final devant une seule consonne ou pause selon le locuteur individuel

\begin{tabular}{|c|c|c|c|}
\hline locuteur & $\begin{array}{l}\text { taux d'élision devant } \\
\text { une seule consonne }\end{array}$ & $\begin{array}{l}\text { taux d'élision devant } \\
\text { pause }\end{array}$ & $\begin{array}{l}\text { taux d'élision devant } \\
\text { consonne et pause }\end{array}$ \\
\hline $\mathrm{A}$ & $84 \% \quad(31 / 37)$ & $67 \% \quad(4 / 6)$ & $81 \% \quad(35 / 43)$ \\
\hline B (13brp2) & $61 \% \quad(28 / 46)$ & $42 \% \quad(5 / 12)$ & $57 \% \quad(33 / 58)$ \\
\hline $\mathrm{C}$ & $83 \% \quad(19 / 23)$ & $(8 / 13)$ & $(27 / 36)$ \\
\hline $\mathrm{D}$ & $81 \% \quad(13 / 16)$ & $(7 / 19)$ & $(20 / 35)$ \\
\hline E (13apd1) & $54 \% \quad(14 / 26)$ & $62 \% \quad(16 / 26)$ & $(30 / 52)$ \\
\hline $\mathrm{F}$ & $52 \% \quad(15 / 29)$ & $(11 / 21)$ & $(26 / 50)$ \\
\hline G & $55 \% \quad(16 / 29)$ & $(7 / 21)$ & $(23 / 50)$ \\
\hline H (13bpa1) & $84 \% \quad(32 / 38)$ & $82 \% \quad(14 / 17)$ & $(46 / 55)$ \\
\hline I & $73 \% \quad(38 / 52)$ & $(24 / 30)$ & $(48 / 75)$ \\
\hline $\mathrm{J}(13 \mathrm{aas} 1)$ & $77 \% \quad(30 / 39)$ & $(24 / 30)$ & $(54 / 69)$ \\
\hline total & $70 \%(236 / 335)$ & $56 \%(106 / 188)$ & $65 \%(342 / 523)$ \\
\hline Diller (1978:15) & $40 \%(182 / 456)$ & $23 \% \quad(49 / 213)$ & $35 \%(231 / 669)$ \\
\hline Durand et al. (1987 :997) & $19 \% \quad(26 / 140)$ & $(2 / 114)$ & $11 \% \quad(28 / 254)$ \\
\hline $\begin{array}{l}\text { Armstrong et Unsworth } \\
(1999: 140-141)\end{array}$ & $52 \%(149 / 288)$ & $31 \%(142 / 465)$ & $39 \%(291 / 753)$ \\
\hline
\end{tabular}


Le Tableau 2 nous montre aussi qu'il y a des différences importantes entre les locuteurs individuels de notre corpus, mais que ces différences ne correspondent pas directement à l'âge des locuteurs. Le Locuteur G, âgé de 56 ans, a le taux d'élision le plus bas (46\%), tandis que le Locuteur A, âgé de 45 ans, a le taux d'élision le plus élevé (81\%). Mais le Locuteur $\mathrm{J}$, le plus âgé à 73 ans, a un taux d'élision relativement élevé (78\%) par rapport au Locuteur B, le plus jeune avec le Locuteur A à 45 ans, qui a un taux d'élision relativement bas (57\%). Pustka (2007:86) a trouvé une corrélation un peu plus directe entre l'âge des locuteurs et l'élision du schwa. Le taux d'élision des locuteurs âgés de 54 ans et plus est de $12 \%$ ou moins, avec l'exception d'une locutrice de 65 ans qui affiche un taux d'élision de $61 \%$. Chez les jeunes de 28 ans et moins le taux d'élision est de $39 \%$ ou plus, sauf pour une locutrice de 28 ans qui affichent un taux d'élision de 30\%. Pourtant Durand et al. (1987 :997) ont montré que le taux d'élision du schwa dans leur corpus correspondait parfaitement à l'âge des locutrices, les plus jeunes ayant un taux d'élision plus élevé. Comme mentionné ci-dessus, en fin de mot devant consonne la locutrice de 85 ans affiche un taux d'élision de $0 \%$, n'ayant fait aucune élision, celle de 67 ans un taux d'élision de 4\%, celle de 36 ans de 17\%, et la plus jeune élide $48 \%$ des schwas possibles. Il y a certainement une différence de taux d'élision entre les générations dans l'étude de Durand et al. (1987) qu'on ne trouve pas entre les locuteurs de notre corpus ni de la même façon dans le corpus de Pustka (2007 :86). Peut-être la différence d'âge entre nos locuteurs (entre 45 et 73 ans) n'est pas assez grande ou peut-être d'autres facteurs, comme le lieu d'origine des locuteurs et leur réseau social, affectent leur taux d'élision du schwa. Pustka (2007:86) propose, par exemple, que le bas taux d'élision de la jeune locutrice de 28 ans est dû à sa fidélité à sa région et que le haut taux d'élision de la dame de 65 ans s'explique par son contact avec des locuteurs des autres régions. Les locutrices dans l'étude de Durand et al. (1987) venaient de la même famille et étaient donc à part l'âge plus similaires que les nôtres et ceux dans l'étude de Pustka.

Nous avons choisi de considérer d'abord l'effet de trois groupes de facteurs sur l'élision du schwa, à savoir la consonne individuelle précédente, la consonne individuelle suivante, et la fréquence de la lexie qui contient le schwa définie selon son nombre d'occurrences dans notre corpus de 1 à 29 . Le Tableau 3 présente les résultats pour ces groupes de facteurs d'une analyse multivariée par le biais du logiciel GoldVarb X. Cette analyse est conçue pour déterminer si l'effet d'un certain groupe de facteurs, comme la consonne précédente, est significatif et surtout pour déterminer l'ordre relatif de l'importance des groupes de facteurs significatifs, par exemple, si la consonne précédente a un plus grand effet que la suivante. À l'intérieur de chaque groupe de facteurs, le poids du facteur permet de mettre les facteurs en ordre selon la force de leur effet. Plus le poids du facteur s'approche de 1,00 plus le facteur favorise la variante en question et plus il s'approche de 0,00 plus il la défavorise. Par exemple, la consonne qui favorise le plus l'élision du schwa dans le Tableau 3 est le /R/ dont le poids du facteur est 0,759 , et celle qui la défavorise le plus est le $/ \mathrm{p} /$, dont le poids du facteur est 0,112 seulement.

Les résultats dans le Tableau 3 montrent que, quand les consonnes environnantes et la fréquence de la lexie sont considérées de la façon la plus individuelle, seul l'effet de la consonne précédente est significatif. En termes généraux nous voyons que les consonnes précédentes plus faibles, comme les liquides $/ \mathrm{R} /$ et $/ \mathrm{l} /$, la nasale $/ \mathrm{n} /$ et la semi-voyelle $/ \mathrm{j} /$, favorisent l'élision du schwa plus que les consonnes précédentes plus fortes, comme /p/ et /t/. Mais l'ordre de taux d'élision du schwa du plus haut au plus bas selon la consonne précédente ne suit pas parfaitement la force articulatoire de la consonne. On s'attendrait à ce que les fricatives favorisent l'élision plus que toutes les occlusives, mais même si on écarte les occlusives $/ \mathrm{k} /$ et $/ \mathrm{d} /$ à cause du petit nombre d'occurrences comme on a déjà dû exclure le $/ \mathrm{b} /$ et le $/ \mathrm{g} /$, on voit tout de même que la fricative $/ \mathrm{z} /$ défavorise l'élision tout autant que l'occlusive /t/. En plus, on s'attendrait à ce que toutes les nasales favorisent l'élision plus que les fricatives, mais les fricatives $/ 3 /$ et $/ \mathrm{s} /$ favorisent l'élision plus que la nasale $/ \mathrm{m} /$. Il est intéressant de noter à cet égard que Walker (1996:216-218) trouve, dans son étude de la stabilisation du schwa d'après la prononciation des lexies fournie par quatre dictionnaires de prononciation, que le schwa est le plus souvent variable et donc le plus susceptible d'élision après /3/,/s/, / / / et /R/ (et /n/ avec deux lexies seulement). Il conclut aussi, comme nous le voyons dans le 
Tableau 3, que les consonnes qui suivent le schwa n'ont aucun effet sur sa prononciation. Comme mentionné ci-dessus, Delattre $(1949 \mathrm{~b}: 44)$ a proposé l'ordre suivant des consonnes précédant le schwa dans un monosyllabe allant du plus grand effet sur l'élision au plus faible : /3/, /s/, /1/, /n/, $/ \mathrm{m} /, / \mathrm{d} /, / \mathrm{t} /, / \mathrm{k} /$. Alors, Delattre pour les monosyllabes et Walker pour la syllabe initiale sont d'accord que $/ 3 /$ et $/ \mathrm{s} /$ favorisent l'élision du schwa. Nos résultats montrent aussi, comme le suggère Walker, que le /R/ favorise l'élision. Dans notre corpus /3/ et /s/ favorisent l'élision du schwa plus que les autres fricatives, mais pas dans un sens absolu puisque leur poids du facteur est juste en dessous de 0,500 .

Tableau 3 : Analyse multivariée des consonnes environnantes individuelles et de la fréquence de la lexie sur l'élision du schwa final

\begin{tabular}{|c|c|c|c|}
\hline & poids du facteur & $\%$ d'élision & $\mathrm{N}$ \\
\hline consonne précédente & \multicolumn{3}{|c|}{ probabilité $\log -172,160$, significativité 0,006} \\
\hline$/ \mathrm{R} /$ & 0,759 & 89 & 56 \\
\hline$/ 1 /$ & 0,700 & 86 & 43 \\
\hline$/ \mathrm{k} /$ & 0,570 & 79 & 9 \\
\hline$/ \mathrm{n} /$ & 0,567 & 78 & 49 \\
\hline$/ \mathrm{j} /$ & 0,532 & 75 & 8 \\
\hline$/ 3 /$ & 0,496 & 72 & 18 \\
\hline$/ \mathrm{s} /$ & 0,481 & 71 & 31 \\
\hline$/ \mathrm{m} /$ & 0,441 & 68 & 37 \\
\hline$/ \mathrm{d} /$ & 0,336 & 57 & 7 \\
\hline$/ \mathrm{n} /$ & 0,275 & 50 & 4 \\
\hline$/ \mathrm{v} /$ & 0,202 & 40 & 10 \\
\hline$/ \mathrm{t} /$ & 0,174 & 36 & 28 \\
\hline $\mid \mathrm{z} /$ & 0,170 & 35 & 20 \\
\hline$/ \mathrm{p} /$ & 0,112 & 25 & 4 \\
\hline gamme & 64,7 & & \\
\hline \multicolumn{4}{|c|}{ probabilité $\log -191,072$, significativité 0,726} \\
\hline gamme & 45,0 & & \\
\hline \multicolumn{4}{|c|}{ probabilité $\log -192,381$, significativité 0,825} \\
\hline gamme & 30,2 & & \\
\hline
\end{tabular}

Les exclusions dans le Tableau 3 comprennent une occurrence de la consonne précédente /b/ et deux de la consonne / $/$ / après lesquelles le schwa est élidé, une occurrence de la consonne précédente /f/ après laquelle le schwa est prononcé, 5 occurrences de /g/ comme consonne précédente puisque le segment suivant est toujours une pause, contexte que nous avons exclu de cette analyse, et les 7 occurrences de la lexie grand-mère qui ne se prononcent jamais avec le schwa final.

Nous ne nous attendrions pas à trouver une correspondance exacte entre l'ordre des consonnes proposé par Walker et Delattre et nos résultats, surtout parce que l'effet des consonnes précédentes proposé par Walker est basé sur des dictionnaires de prononciation et celui de Delattre est basé sur son intuition. Le nôtre est basé par contre sur des tests empiriques menés sur la prononciation dans des conversations spontanées. Il est vrai aussi que la position du schwa dans le mot et les variétés de français sont différentes dans les trois études. Il semble pourtant qu'une consonne faible précédente favorise l'élision du schwa et dans les monosyllabes et dans la position finale de mot que que quelques-unes de ces mêmes consonnes favorisent l'élision variable du schwa en syllabe initiale de mot. Nous tirons donc la conclusion de ces premiers résultats que la consonne précédente individuelle a un effet important sur l'élision du schwa, effet qui semble dépendre en partie de la force de la consonne. Pour mieux tester cette idée, surtout en vue du petit nombre d'occurrences de schwa après certaines consonnes, dans l'analyse suivante nous regrouperons les consonnes selon le 
mode d'articulation, le point d'articulation, et le voisement pour voir si les consonnes ainsi regroupées ont un effet sur l'élision du schwa. Ce regroupement rappelle la décision de Malécot (1976:99) de regrouper les consonnes selon le mode d'articulation comme occlusives, fricatives et /1/ vu le petit nombre d'exemples du phénomène dans la conversation spontanée.

Le Tableau 3 montre en plus que ni la consonne suivante ni la fréquence de la lexie n'a d'effet significatif sur l'élision du schwa, donc nous n'avons pas reproduit dans le tableau les résultats pour ces facteurs. Mais le manque de significativité pour ces facteurs ne veut pas dire qu'ils n'ont aucun effet sur l'élision du schwa. Il est possible que la consonne suivante ait un effet mais qui est plus petit que celui de la consonne précédente, donc nous considérerons dans des analyses subséquentes l'interaction entre la consonne précédente et la consonne suivante. La fréquence de la lexie a été définie de façon aussi individuelle que possible pour cette analyse, comme pour les consonnes, puisque nous avons soumis à l'analyse multivariée les lexies avec le même nombre d'occurrences de 1 à 29 dans des catégories séparées. Malgré le manque de significativité pour la fréquence de la lexie, nous voyons que les lexies les plus fréquentes ont le plus haut taux d'élision. Parmi les 164 lexies différentes dans le corpus qui contiennent un schwa en fin de mot devant consonne, seulement 9 apparaissent 6 fois ou plus. Parmi elles, six ont un taux d'élision supérieur à la moyenne de $70 \%$ devant consonne : dire $(75 \%, 9 / 12)$, une $(81 \%, 17 / 21)$, pense $(83 \%, 5 / 6)$, et surtout faire $(89 \%, 8 / 9)$, elle $(92 \%, 12 / 13)$ et grand-mère $(100 \%, 7 / 7)$. Les trois lexies fréquentes qui ont un taux d'élision inférieur à la moyenne sont comme à $64 \%(9 / 14)$, village à $67 \%(6 / 9)$ et même à $69 \%$ (9/13). On remarque aussi que les lexies les plus fréquentes avec le plus haut taux d'élision sont aussi celles qui finissent par /R/,/1/, ou /n/ et donc elles contribuent au haut taux d'élision après ces consonnes.

Ayant vu l'effet des consonnes et des lexies individuelles sur l'élision du schwa, il convient maintenant de voir l'effet possible des groupes de consonnes ou de lexies. Le Tableau 4 montre les résultats pour les consonnes précédentes et suivantes regroupées selon leur mode d'articulation, point d'articulation et voisement et l'effet de la fréquence des lexies. Cette fois-ci nous avons formé trois groupes de fréquence au lieu de 15 en regroupant les lexies avec 1 à 2 occurrences, celles avec 3 à 5 occurrences, et celles avec 6 occurrences ou plus. Comme nous avons prédit à partir des résultats pour les consonnes individuelles, les résultats pour la consonne précédente sont toujours significatifs pour toutes leurs caractéristiques, notamment le mode d'articulation, le point d'articulation, et le voisement. Les liquides, les vélaires, et les consonnes voisées favorisent l'élision du schwa tandis que les fricatives et occlusives, les bilabiales et labiodentales et les consonnes non voisées défavorisent son élision. Parmi ces trois traits, c'est le mode d'articulation qui a été choisi par Goldvarb X comme le facteur le plus important, ce qui est reflété dans sa significativité de 0,000, sa probabilité log le plus proche de zéro, et aussi sa plus grande gamme de 49,4, ce qui est la différence entre le poids du facteur le plus élevé pour le groupe de facteurs $(0,740$ pour les liquides) et le poids de facteur le plus bas (0,246 pour les occlusives). Cela renforce ce que nous avons vu dans le Tableau 3 pour les consonnes individuelles : un /R/ ou /1/ précédent favorise l'élision du schwa. Nous voyons en plus que le point d'articulation bilabial ou labiodental défavorise l'élision du schwa ce qui expliquerait le faible taux d'élision après $/ \mathrm{m} /$ par rapport à $/ \mathrm{n} /$ et de $/ \mathrm{v} /$ par rapport à $/ 3 /$.

La fréquence de la lexie contenant le schwa n'était pas significative quand chaque nombre d'occurrences a été considéré séparément dans le Tableau 3, mais quand les nombres d'occurrences sont codés en trois groupes la fréquence de la lexie devient significative. Nous observons qu'une lexie plus fréquente favorise l'élision du schwa en fin de mot dans notre corpus comme elle le fait aussi pour le schwa en position initiale de mot dans le corpus parisien de Hansen (1994:39-41). Il est intéressant de noter que, de la même façon que dans notre étude, la fréquence n'était pas significative dans l'étude de Hansen (1994) quand elle avait fait des distinctions de fréquence plus fines, mais qu'elle l'était quand elle a regroupé les fréquences pour comparer les mots d'une occurrence et ceux de 2 à 9 occurrences avec les mots de 10 occurrences ou plus. Ces résultats confirment une tendance qui remonte jusqu'à Dell (1973:230, cité par Hansen 1994 :30 et Racine et 
Grosjean 2002 :311) qui a proposé que les mots peu courants ont tendance à garder le schwa. Cette tendance pour l'élision du schwa suit le principe encore plus général de Zipf (1949) qui maintient que plus un mot est fréquent, plus il est court. Il est intéressant que l'effet de la fréquence soit le même pour le schwa et en position finale et en position initiale.

Tableau 4 : Analyse multivariée des consonnes environnantes selon leur mode d'articulation, point d'articulation et voisement et de la fréquence de la lexie sur l'élision du schwa final

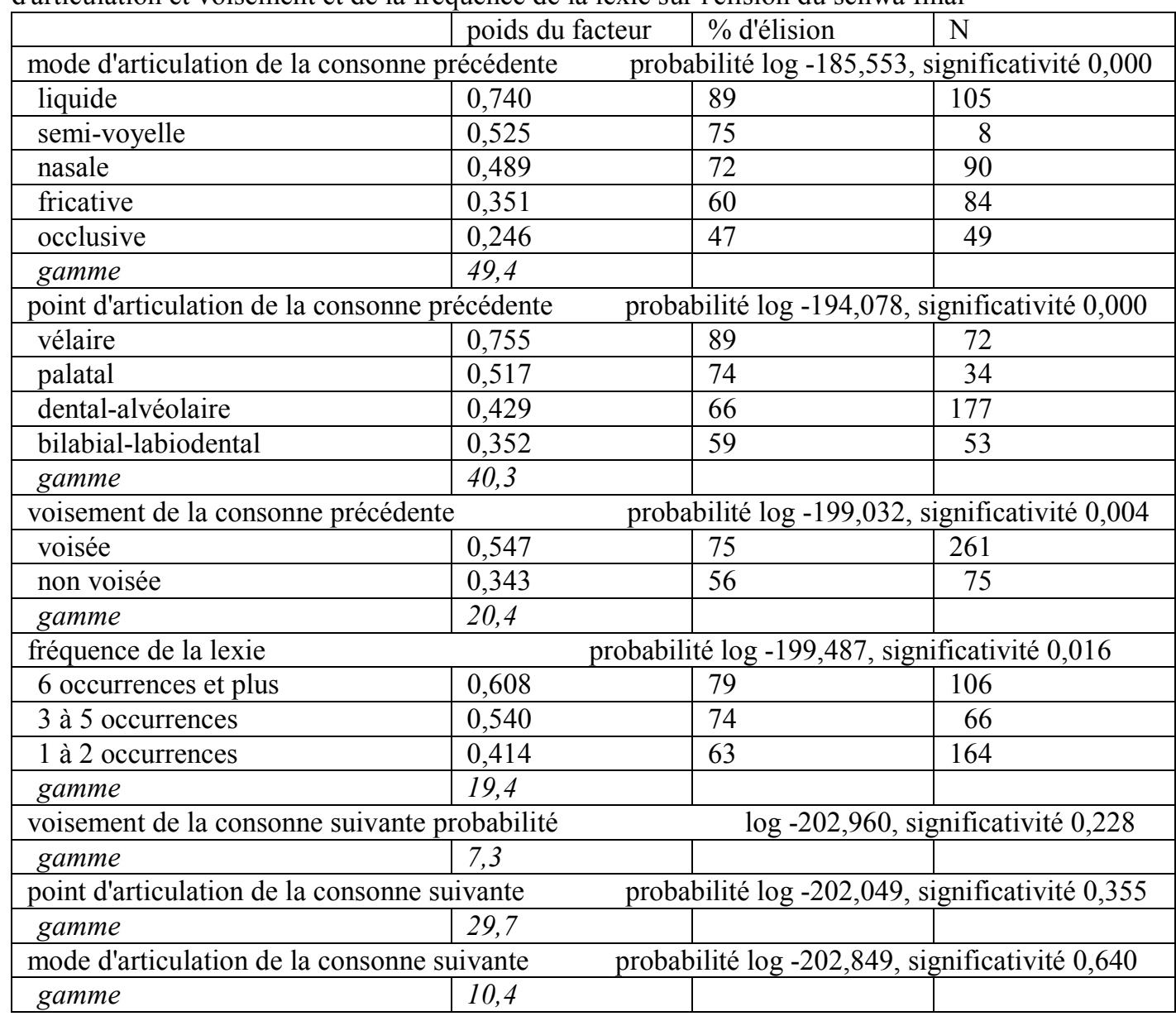

Parmi les facteurs linguistiques testés nous avons vu jusqu'ici que la consonne précédente a le plus grand effet sur l'élision du schwa et que la fréquence de la lexie a aussi un effet mais moins important que celui de la consonne précédente. Nous n'avons pas trouvé d'effet significatif de la consonne suivante sur l'élision du schwa mais il est possible tout de même qu'il y ait une interaction entre la consonne précédente et la suivante. Pour mettre cette idée à l'épreuve, nous avons formé des facteurs basés sur cette interaction dont les résultats sont présentés dans le Tableau 5. Nous avons testé ces facteurs contre la fréquence de la lexie pour continuer à voir l'importance relative des facteurs linguistiques. Pour le voisement, par exemple, nous avons considéré l'effet des combinaisons suivantes: consonne précédente voisée et consonne suivante voisée, consonne précédente voisée et consonne suivante non voisée, consonne précédente non voisée et consonne suivante voisée et finalement les deux consonnes non voisées. Nous avons choisi de regrouper les 8 occurrences de la semi-voyelle /j/ comme consonne précédente avec les nasales puisque le poids de facteur pour les semi-voyelles était le plus proche de celui pour les nasales, comme nous avons vu dans le Tableau 4. 
Nous voyons dans le Tableau 5 des résultats significatifs pour trois groupes de facteurs: la fréquence de la lexie, le voisement des consonnes environnantes, et le mode d'articulation des consonnes environnantes. Nous avons déjà vu dans le Tableau 4 que la fréquence de la lexie était significative mais moins importante comme facteur que la consonne précédente. Quand nous l'avons testée de nouveau ici contre les facteurs pour l'interaction entre les consonnes environnantes, elle en sort comme le facteur le plus important. En ce qui concerne l'interaction entre les consonnes environnantes, l'élision du schwa est favorisée entre deux consonnes voisées mais défavorisée entre deux consonnes non voisées. La transition de consonne voisée à consonne voisée se fait plus souvent donc sans le schwa mais le schwa apparaît plus souvent dans la transition entre deux consonnes non voisées. Dans les résultats pour le mode d'articulation nous observons le même ordre général pour la consonne précédente allant du mode qui favorise l'élision le plus au mode qui la favorise le moins, à savoir liquide, nasale, fricative, occlusive. À l'intérieur de chaque mode d'articulation de la consonne précédente, nous trouvons que le schwa est favorisé en général quand la consonne suivante a le même mode d'articulation que la précédente. Il paraît que le schwa sert à séparer des consonnes ayant le même mode d'articulation. Nous constatons finalement que l'interaction entre le point d'articulation des consonnes environnantes n'a pas d'effet significatif sur l'élision.

Tableau 5 : Analyse multivariée de l'interaction entre les consonnes environnantes selon leur mode d'articulation, point d'articulation et voisement et de la fréquence de la lexie

\begin{tabular}{|c|c|c|c|}
\hline & poids du facteur & \% d'élision & $\mathrm{N}$ \\
\hline \multicolumn{4}{|c|}{ probabilité $\log -195,305$, significativité 0,022} \\
\hline 6 occurrences et plus & 0,604 & 79 & 104 \\
\hline 3 à 5 occurrences & 0,541 & 74 & 62 \\
\hline 1 à 2 occurrences & 0,417 & 64 & 162 \\
\hline gamme & 18,7 & & \\
\hline \multicolumn{4}{|c|}{ voisement des consonnes environnantes } \\
\hline voisée-voisée & 0,587 & 78 & 125 \\
\hline voisée-non voisée & 0,498 & 71 & 131 \\
\hline non voisée-voisée & 0,406 & 63 & 40 \\
\hline non voisée-non voisée & 0,304 & 52 & 30 \\
\hline gamme & 28,3 & & \\
\hline \multicolumn{4}{|c|}{ mode d'articulation des consonnes environnantes probabilité $\log -179,859$, significativité 0,045} \\
\hline liquide-fricative & 0,807 & 92 & 25 \\
\hline liquide-occlusive & 0,745 & 89 & 54 \\
\hline liquide-nasale & 0,718 & 88 & 8 \\
\hline liquide-liquide & 0,593 & 80 & 15 \\
\hline nasale-occlusive & 0,542 & 77 & 51 \\
\hline nasale-liquide & 0,542 & 77 & 17 \\
\hline fricative-nasale & 0,422 & 67 & 3 \\
\hline nasale-nasale & 0,422 & 67 & 3 \\
\hline fricative-occlusive & 0,385 & 63 & 57 \\
\hline nasale-fricative & 0,354 & 60 & 25 \\
\hline occlusive-fricative & 0,305 & 55 & 11 \\
\hline occlusive-occlusive & 0,267 & 50 & 22 \\
\hline fricative-fricative & 0,267 & 50 & 14 \\
\hline fricative-liquide & 0,226 & 44 & 9 \\
\hline occlusive-liquide & 0,215 & 43 & 14 \\
\hline gamme & 59,2 & & \\
\hline \multicolumn{4}{|c|}{ point d'articulation des consonnes environnantes $\quad$ probabilité log $-187,391$, significativité 0,351} \\
\hline gamme & 49,8 & & \\
\hline
\end{tabular}


Nous avons dû exclure du Tableau 5 les deux occurrences d'occlusive précédente suivie par nasale puisque le schwa a été prononcé dans les deux cas. Le schwa a toujours été élidé dans les trois occurrences de consonne palatale suivie par palatale et les deux cas de consonne bilabiale suivie par palatale, donc ces exemples ont été également exclus.

En général l'importance du voisement et du mode d'articulation de la consonne précédente est moins forte quand elle est combinée avec la consonne suivante. On voit que la significativité pour le voisement et le mode d'articulation est plus haute, c'est-à-dire moins significative, pour l'interaction entre les consonnes que pour la consonne précédente seule, présentée dans le Tableau 4. La significativité pour le voisement pour l'interaction est 0,023 contre 0,004 pour la consonne précédente toute seule et 0,045 contre 0,000 pour le mode d'articulation.

Ces résultats nous font penser que la considération de la consonne suivante ne fait que diminuer l'effet du voisement et du mode d'articulation de la consonne précédente. Pour isoler l'effet de la consonne suivante de celui de la consonne précédente, nous avons testé les facteurs significatifs du Tableau 5 pour chaque mode de consonne précédente séparément. Cela veut dire que nous avons soumis à l'analyse multivariée l'effet de la fréquence de la lexie et de l'interaction entre le voisement de la consonne précédente et la suivante et entre le mode d'articulation de ces deux consonnes pour les consonnes précédentes liquides, nasales, fricatives, et occlusives à tour de rôle. Nous voulions voir de cette façon si la consonne suivante aurait un effet sur l'élision du schwa pour les consonnes précédentes qui avaient le même mode d'articulation. Une fois éliminée la différence de mode d'articulation entre les consonnes précédentes, il n'y avait plus aucune différence significative entre les autres facteurs, ni la fréquence de la lexie, ni le voisement, ni le mode d'articulation. Cela renforce notre soupçon que c'est plutôt la consonne précédente qui affecte l'élision du schwa et non pas l'interaction entre la consonne précédente et la suivante.

Une autre façon de tester l'interaction possible entre les deux consonnes environnantes est de définir cette interaction en termes de la force articulatoire relative des consonnes, un facteur qui peut être exprimé aussi en termes de la hiérarchie de sonorité. On peut ainsi définir la transition de la consonne précédente à la suivante comme ayant une sonorité descendante, ascendante ou égale. Puisque les consonnes les plus faibles, les liquides et les nasales, favorisent le plus l'élision du schwa, il semble probable qu'une consonne précédente plus faible que la suivante favorisera l'élision et qu'une consonne précédente plus forte la défavorisera. De tels résultats soutiendraient ce que Delattre $(1949 \mathrm{~b}: 44)$ a proposé et ce que Malécot $(1976: 99)$ a trouvé pour le schwa des monosyllabes en début d'énoncé, c'est-à-dire qu'une première consonne plus faible que la seconde favorise l'élision du schwa. Mais de tels résultats seraient le contraire de ce que Hansen (1994) et Racine et Grosjean (2002) ont trouvé pour le schwa en syllabe initiale de mot. Si on formule cette idée en termes de la hiérarchie de sonorité on dira qu'une sonorité descendante favorisera l'élision du schwa. Pour cette analyse nous avons déterminé la force de la consonne et sa sonorité selon le mode d'articulation sans considérer le voisement des occlusives et fricatives. Cette décision se doit en partie au petit nombre d'occurrences de quelques occlusives, mais elle est justifiée en plus par le fait que Clements (1990:292) définit la hiérarchie de sonorité en termes de quatre traits majeurs de classe ('major class features'), à savoir les obstruantes, les nasales, les liquides, et les semi-voyelles sans tenir compte de leur voisement. Dans notre classification, nous avons choisi de distinguer entre occlusives et fricatives pour la catégorie obstruantes et de combiner les quelques occurrences de semi-voyelles avec les nasales. Si la consonne précédente est plus forte que la suivante, nous classons la sonorité comme ascendante, selon l'échelle de sonorité suivante qui va de la sonorité la plus basse à la plus haute : occlusives, fricatives, nasales et semi-voyelle $/ \mathrm{j} /$, et liquides. Si la consonne précédente est plus faible que la suivante, nous classons la sonorité comme descendante. $\mathrm{Si}$ les deux consonnes ont la même sonorité nous classons la sonorité comme égale. Notre hypothèse est que le schwa en fin de mot suivra la tendance que Malécot (1976:99) a identifiée pour le schwa des monosyllabes, notamment qu'une première consonne plus forte que la seconde défavorisera l'élision du schwa et inversement qu'une consonne précédente plus faible que la 
suivante favorisera l'élision du schwa. En termes de la hiérarchie de sonorité, une sonorité ascendante défavorisera l'élision du schwa et une sonorité descendante la favorisera.

Tableau 6: Analyse multivariée de l'effet de la sonorité relative de la consonne précédente par rapport à la suivante sur l'élision du schwa final

\begin{tabular}{|l|l|l|l|}
\hline & poids du facteur & \% d'élision & N \\
\hline sonorité descendante & 0,575 & 77 & 226 \\
\hline sonorité égale & 0,353 & 57 & 54 \\
\hline sonorité ascendante & 0,344 & 56 & 55 \\
\hline gamme & 23,1 & & \\
\hline \multicolumn{2}{|r|}{ probabilité log $-196,412$, significativité 0,001} \\
\hline
\end{tabular}

Les résultats pour la sonorité présentés dans le Tableau 6 montrent que l'élision du schwa méridional en fin de mot est favorisé par une sonorité descendante, comme dans les séquences dire que, village de, et seule chose, où la force de la consonne qui précède le schwa est plus faible que celle qui le suit. Une sonorité égale, comme dans je ne regrette pas et c'est grave ça, et une sonorité ascendante, comme dans église romane et service militaire, défavorisent l'élision du schwa. Il paraît donc que les locuteurs ont une plus grande tendance à prononcer le schwa quand il est entre deux consonnes avec le même degré de sonorité ou entre une consonne plus forte suivie par une consonne plus faible. Pour voir si cette corrélation dépendait surtout de la consonne précédente et non pas de l'interaction entre les deux consonnes, nous avons testé l'effet de la sonorité pour chaque mode d'articulation de la consonne précédente. Comme pour l'interaction entre le voisement de la consonne précédente et la suivante et aussi pour le mode d'articulation entre ces deux consonnes, nous avons trouvé que la sonorité n'était plus significative quand chaque mode d'articulation de la consonne précédente était testé séparément. Il paraît encore une fois que c'est la consonne précédente qui a le plus grand effet sur l'élision du schwa. La sonorité descendante favorise l'élision du schwa final puisque les consonnes qui favorisent le plus l'élision du schwa final sont les liquides et nasales qui sont aussi les consonnes sont les plus faibles et les plus sonores sur la hiérarchie de la sonorité.

\section{Conclusion}

Cette étude est la première à tester l'effet des facteurs linguistiques sur la prononciation variable du schwa en fin de mot chez des locuteurs méridionaux selon l'approche variationniste labovienne. L'analyse multivariée par le biais de GoldVarb X montre que la consonne précédente a le plus grand effet sur l'élision du schwa et que la fréquence de la lexie a un effet secondaire. La consonne suivante quand elle était testée séparément n'a jamais eu aucun effet, mais l'interaction entre les consonnes environnantes s'est avérée significative pour le mode d'articulation et le voisement de ces consonnes et aussi pour leur force selon la hiérarchie de la sonorité. Nous soupçonnons pourtant que ces effets soient dus plutôt à l'effet de la consonne précédente plutôt qu'à une vraie interaction entre les consonnes environnantes.

En ce qui concerne les consonnes précédentes individuelles, ce sont les liquides /R/ et /1/ qui favorisent fortement l'élision du schwa, avec un poids de facteur de 0,759 et 0,700 respectivement. $\mathrm{Le} / \mathrm{n} /$ la favorise aussi mais à un moindre degré ayant un poids de facteur de 0,567 . Les consonnes qui défavorisent l'élision du schwa sont surtout le /z/ (avec un poids du facteur de 0,170), le /t/ $(0,174)$, et le $/ \mathrm{v} /(0,202)$. Les consonnes $/ 3 /(0,496)$, $/ \mathrm{s} /(0,481)$ et $/ \mathrm{m} /(0,481)$ ne défavorisent l'élision que faiblement et le nombre d'occurrences dans le corpus des consonnes précédentes $/ \mathrm{k} /$, $/ \mathrm{j} /, / \mathrm{d} /, / \mathrm{n} /$, et $/ \mathrm{p} /$ est trop petit pour en tirer des conclusions fiables. Quand les consonnes précédentes sont regroupées selon le mode d'articulation, ce sont surtout les liquides qui favorisent l'élision, comme nous avons déjà vu pour $/ \mathrm{R} /$ et $/ \mathrm{l} /$. Les nasales défavorisent un peu l'élision et les fricatives et surtout les occlusives la défavorisent plus fortement. Le point d'articulation vélaire favorise fortement l'élision, sans doute à cause $\mathrm{du} / \mathrm{R} /$, le palatal la favorise un peu moins et le point 
d'articulation bilabial-labiodental défavorise l'élision. Nous avons trouvé aussi une corrélation entre le voisement de la consonne précédente et l'élision de façon à ce que les consonnes voisées favorisent l'élision tandis que les non voisées la défavorisent. En plus de l'effet très net de la consonne précédente sur l'élision du schwa, nous avons trouvé une corrélation entre la fréquence de la lexie et l'élision du schwa. Une lexie plus fréquente, qui apparaît 3 fois ou plus dans le corpus, favorise l'élision tandis qu'une lexie qui n'apparaît qu'une ou deux fois la défavorise. Cela suit le principe général de Zipf (1949) que plus un mot est fréquent plus il sera court.

Cette analyse a aussi dégagé l'effet d'une possible interaction entre la consonne précédente et la consonne suivante. Quand les deux consonnes sont voisées le schwa est plus souvent élidé mais il est plus souvent prononcé quand les deux consonnes sont non voisées. Les combinaisons selon le mode d'articulation respectent l'ordre que nous avons vu ci-dessus pour la consonne précédente de liquide, nasale, fricative et occlusive en commençant par le mode qui favorise davantage l'élision. À l'intérieur de chaque mode d'articulation de la consonne précédente, nous trouvons qu'une consonne suivante avec le même mode que la précédente favorise moins l'élision du schwa que les autres modes de la consonne suivante, ce qui fait penser que le schwa puisse servir à séparer deux consonnes similaires. Une autre façon de tester l'interaction entre les consonnes environnantes était de voir si la sonorité montait ou descendait entre la consonne précédente et la suivante. Nous voyons qu'une sonorité descendante entre ces deux consonnes favorise l'élision du schwa et inversement qu'une sonorité égale ou ascendante favorise son maintien. Il reste à revoir dans de futures études pourtant si cet effet se doit surtout à la forte tendance des liquides et nasales à favoriser l'élision du schwa ou s'il y a une vraie interaction entre les consonnes environnantes.

La comparaison de nos résultats avec ceux des études sur d'autres positions du schwa dans d'autres variétés du français soulève une question théorique importante: Pourquoi est-ce que la prononciation variable du schwa serait affectée différemment par les consonnes environnantes selon sa position dans le mot? Nous avons trouvé, par exemple, que plus la première consonne est faible par rapport à la seconde plus le schwa est élidé. Ce comportement variable du schwa suit celui que Delattre (1949b) et Malécot (1976) ont décrit pour le schwa des monosyllabes mais il va à l'encontre de ce que Hansen (1994) a trouvé en syllabe initiale de mot qui ne commence pas par re-chez des locuteurs parisiens et ce que Racine et Grosjean (2002) ont trouvé en syllabe initiale chez des locutrices suisses romandes. Une possibilité que nous mettrons à l'épreuve dans une future étude, dépendrait de la séquence des monosyllabes, syllabes en fin de mot, et syllabes initiales de mot dans la chaîne parlée. Puisque les syllabes initiales de mot suivent souvent les monosyllabes et les syllabes en fin de mot, les tendances inverses pourraient favoriser le maintien du schwa dans l'une ou l'autre position pour que deux schwas de suite ne s'élident pas. Pour mieux comprendre ce phénomène, il serait nécessaire de considérer le taux d'élision du schwa dans toutes les positions du mot chez les locuteurs de notre corpus, c'est-à-dire dans les monosyllabes et les syllabes initiales de mot en plus de la position finale, pour voir si l'effet des consonnes environnantes diffère selon sa position dans le mot dans la parole de nos locuteurs.

L'analyse présentée ici de quelques facteurs linguistiques ne constitue qu'une première tentative d'explication de la variabilité du schwa méridional en fin de mot. Il faudrait dans l'avenir considérer d'autres facteurs linguistiques en plus des consonnes environnantes et la fréquence de la lexie, surtout pour expliquer les cas où un seul locuteur varie sa prononciation du schwa final dans le même mot. Il y a en tout dans le corpus 23 mots qui sont prononcés de façon variable devant consonne par le même locuteur. Un exemple est le mot comme dans comme ça, cité au début de l'article, prononcé par le Locuteur B; d'autres mots dont la prononciation varie chez le même locuteur sont contrôle et grave dans la parole du Locuteur $\mathrm{G}$ et église, même, romane, stage, une et village dans la parole du Locuteur I. Quelques facteurs possibles à tester sont le débit, le nombre de syllabes qui suivent le schwa dans le même groupe de souffle, et le désir de faire ressortir l'idée exprimée par le mot terminant par schwa. On pourrait supposer qu'un débit rapide favoriserait l'élision et qu'un débit lent favoriserait le maintien du schwa. Racine et Grosjean (2002 :318-319) ont trouvé qu'il existe une corrélation entre la vitesse d'articulation et l'élision mais disent que 
«d'autres possibilités sont également présentes ». L'idée que le nombre de syllabes suivant le schwa puisse affecter sa prononciation est basée sur l'observation de Léon (1966, cité par Spence 1982 :4 et Morin 1983 :76) que le schwa du mot porte dans porte-feuille est toujours prononcé mais qu'il est le plus souvent élidé dans porte-bébé. On supposerait donc que l'élision su schwa serait favorisé quand deux ou plusieurs syllabes le suivraient dans le groupe de souffle que quand une seule syllabe le suivrait. Finalement, comme la première auteure de cette étude a proposé pour la liaison (Ranson $2008: 1681$ ), un locuteur pourrait prononcer le schwa pour mettre en relief l'idée exprimée par le mot qui le contient. Pulgram (1961:306) fait référence à cette possibilité quand il mentionne le style, qui d'après lui comprend la vitesse, l'emphase et l'intention, comme un facteur possible dans la prononciation du schwa. Racine et Grosjean (2002 :319-320) n'ont pas trouvé de corrélation entre l'importance discursive et la réalisation du schwa mais ils ont déterminé cette importance en demandant à d'autres locuteurs de souligner les mots qu'ils trouvaient importants dans le texte écrit et non pas en déterminant ce que les locutrices elles-mêmes voulaient faire ressortir. Pour qu'il y ait une corrélation entre la mise en relief et la réalisation du schwa, il faudrait qu'un locuteur s'en serve pour faire ressortir un certain mot et donc une certaine idée. Nous savons donc que la consonne précédente et la fréquence de la lexie qui contient le schwa affecte son élision. Dans l'avenir nous testerons de nouveau l'interaction entre les consonnes environnantes pour mieux déterminer l'effet de ce facteur sur l'élision et nous considérerons d'autres facteurs linguistiques pour mieux comprendre la variabilité du schwa final, l'aspect jusque là méconnu d'un phénomène bien connu des méridionaux.

\section{Références bibliographiques}

Armstrong, N. et Unsworth, S. (1999). Sociolinguistic variation in southern French schwa. Linguistics, 37, $127-156$

Clements, G. N. (1990). The role of the sonority cycle in core syllabification. In Kingston, J. et Beckman, M. E. (éds.), Papers in laboratory phonology 1: between the grammar and physics of speech. Cambridge: Cambridge University Press, 283-333.

Coquillon, A., Di Cristo, A. et Pitermann, M. (2000). Marseillais et Toulousains gèrent-ils différemment leurs pieds? Caractéristiques prosodiques du schwa dans les parlers méridionaux. Actes des XXIIIe Journées d'Étude sur la Parole (JEP), 19-23 juin, Aussois, 89-92.

Dauses, A. (1973). Études sur l'e instable dans le français familier. Tübingen: Niemeyer.

Delattre, P. (1949a). Le Jeu de l'e instable de monosyllabe initial en français. French Review, 22, 455-459.

Delattre, P. (1949b). Le Jeu de l'e instable de monosyllabe initial en français. French Review, 23, 43-47.

Dell, F. (1973). Les Règles et les sons. Introduction à la phonologie générative. Paris: Hermann.

Diller, A-M. (1978). The Mute E as a Sociolinguistic Variable. Montreal Working Papers in Linguistics, 10, $11-21$.

Durand, J. (2009). Essai de panorama critique des accents du midi. In Baronian, L. et Martineau, F. (éds.), Le français, d'un continent à l'autre: Mélanges offerts à Yves Charles Morin. Collection Les Voies du français. Québec: Presses de l’Université Laval, 123-170.

Durand J. et Laks B. (2000). Relire les phonologues du français: Maurice Grammont et la loi des trois consonnes. Langue française, 126, 29-38.

Durand, J., Slater, C. et Wise, H. (1987). Observations on schwa in southern French. Linguistics, 25, 983-1004.

Eychenne, J. (2006). Aspects de la phonologie du schwa dans le français contemporain: optimalité, visibilité prosodique, gradience. Thèse de doctorat, Université de Toulouse-Le Mirail.

Fagyal, Zs. (2000). Le retour du $e$ final en français parisien: changement phonétique conditionné par la prosodie. In Englebert, A. Pierrard, M., Rosier, L. et Van Raemdonck, D. (éds.), Vivacité et diversité de la variation linguistique. Tübingen: Max Niemeyer, III, 151-160. 
Fagyal, Zs., Kibbee, D. et Jenkins, F. (2006). French: A linguistic introduction. Cambridge: Cambridge University Press.

Fouché, P. (1959). Traité de prononciation française, 2e édition. Paris: Klincksieck.

Grammont, M. (1894). Le patois de la Franche-Montagne et en particulier de Damprichard (Franche-Comté). IV: La loi des trois consonnes. Mémoires de la Société de linguistique de Paris, 8, 53-90.

Hansen, A. B. (1994). Étude du E caduc-stabilisation en cours et variations lexicales. Journal of French Language Studies, 4, 25-54.

Hansen, A. B. (1997). Le nouveau e prépausal dans le français parlé à Paris. In Perrot, J. (éd.), Polyphonie pour Iván Fónagy. Paris: Éditions de l'Harmattan, 173-198.

Hansen, A. B. et Hansen, M-B. M. (2002). Le e prépausal et l'interaction. In A. B Hansen, A. B. et Hansen, MB. M. (éds.), Structures linguistiques et interactionnelles dans le français parlé. Actes du Colloque international, Université de Copenhague du 22 au 23 juin 2001 (Études romanes 54). Copenhague: Museum Tusculanum Press, 89-109.

Léon, Pierre. (1966). Apparition, maintien et chute du 'E caduc'. La Linguistique 2, 111-122.

Malécot. A. (1955). The elision of the French mute-e within complex consonantal clusters. Lingua, 5, 44-60.

Malécot, A. (1976). The effect of linguistic and paralinguistic variables on the elision of the French mute-e. Phonetica, 33, 93-112.

Morin, Y.-C. (1983). Quelques observations sur la chute du e muet dans le français régional de Saint-Étienne. La Linguistique 19, 71-93.

Pulgram, E. (1961). French /ə/: Statics and dynamics of linguistic sub-codes. Lingua, 10, 305-325.

Pustka, E. (2007). Phonologie et variétés en contact. Aveyronnais et Guadeloupéens à Paris. Tübingen: Narr.

Pustka, E. (2009). A prototype theoretic model of Southern French. In Beeching, K., Armstrong, N., et Gadet, F. (éds.), Sociolinguistic variation in Contemporary French. Amsterdam/Philadelphia: John Benjamins, 77-94.

Racine, I. et Grosjean, F. (2002). La production du E caduc facultatif est-elle prévisible? Un début de réponse. French Language Studies, 12, 307-326.

Ranson, D. L. (2008). La liaison variable dans un corpus du français méridional: L'importance relative de la fonction grammaticale. In Durand, J., Habert, B., et Laks, B. (éds.), Congrès Mondial de Linguistique Française. Paris: Institut de Linguistique Française, 1669-1683.

Spence, N. C. W. (1982). Another look at the loi des trois consonnes. French Studies, 36, 1-11.

Tagliamonte, S. (2006). Analysing sociolinguistic variation. Cambridge: Cambridge University Press.

Taylor, J. (1996). Sound Evidence: Speech communities and social accents in Aix-en-Provence. Berne: Peter Lang.

Tranel, B. (1987). The Sounds of French: An introduction. Cambridge: Cambridge University Press.

Valdman, A. (1993). Bien Entendu! Introduction à la prononciation française. Englewood Cliffs, NJ: Prentice Hall.

Walker, D. C. (1996). The new stability of unstable -e in French. French Language Studies, 6, 211-229.

Zipf, G. K. (1949). Human behavior and the principle of least effort. Cambridge, MA: Addison-Wesley. 